\title{
Use of Platelet-Rich Plasma in Treating Low Back Pain: A Review of the Current Literature
}

\author{
Mirza Zain Baig ${ }^{1}, U_{m m}$ E Hani Abdullah', Aun Muhammad³ \\ Abeer Aziz ${ }^{4}$, Maryam Jamil Syed ${ }^{5}$, Aneela Darbar ${ }^{6}$ \\ ${ }^{1}$ Department of Surgical Oncology, Dyson Cancer Center, Health Quest Health Systems/Nuvance Health, Poughkeepsie, NY, USA \\ ${ }^{2}$ Weldon School of Biomedical Engineering, Purdue University, West Lafayette, IN, USA \\ ${ }^{3}$ Medical College, Aga Khan University Hospital, Karachi, Pakistan \\ ${ }^{4}$ Department of Surgery, Aga Khan University Hospital, Karachi, Pakistan \\ ${ }^{5}$ Department of Neurology, Aga Khan University Hospital, Karachi, Pakistan \\ ${ }^{6}$ Department of Neurosurgery, Aga Khan University Hospital, Karachi, Pakistan
}

Low back pain (LBP) is a common problem encountered by physicians. It is a considerable cause of morbidity and socioeconomic loss and is one of the most expensive musculoskeletal disorders. Conventional treatments include bed rest, analgesics, therapeutic exercises, lumbar or caudal epidural corticosteroids, and surgery. Several new biological therapies are being investigated for use in LBP and one of these is platelet-rich plasma (PRP). In this article, we summarize the current literature published on PRP concerning its composition, classification, and application in LBP. We believe our review will prove useful to clinicians and academics alike, interested in new developing therapies for LBP.

Keywords: Platelet-rich plasma; Low back pain; Radiculopathy; Epidural

\section{Introduction}

Low back pain (LBP) is a common problem and a significant cause of morbidity and socioeconomic loss to society [1]. It is the second leading cause of disability in American adults and one of the main reasons why people under 45-years limit their physical activity [2-4]. It imposes a considerable disease burden on society and is one of the most expensive musculoskeletal disorders [5].

When assessing patients with LBP, it is important to look for radicular symptoms such as radiating leg pain, sensory deficits, and neurologic lower limb deficits such as motor weakness, and altered reflexes. Many factors contribute to this pathological process. These include disc degeneration leading to herniation, facet arthropathy, ligamentum flavum hypertrophy, ossification of the posterior longitudinal ligament, congenitally narrow neural foramen, ligament laxity, and subluxation [6]. Advancing age contributes to these pathological processes and it is logical to assume that with the increase in life expectancies globally, the incidence of LBP will also increase [7].

Patients with LBP can often be managed conservatively with a combination of pharmacological and nonpharmacological therapies without the need for surgical or inva-

Received May 14, 2019; Revised Sep 24, 2019; Accepted Oct 21, 2019

Corresponding author: Mirza Zain Baig

Department of Surgical Oncology, Dyson Cancer Center, Health Quest Health Systems/Nuvance Health, Poughkeepsie, NY, USA

Tel: +92-1-4694493844, Fax: +92-1-4694493844, E-mail: mzainbaig@gmail.com 
sive procedures [2]. Conservative treatment includes bed rest, analgesic medications such as nonsteroidal anti-inflammatory drugs (NSAIDs) and acetaminophen, muscle relaxants, oral or parenteral steroids, and opioids as well as therapeutic exercises [1,2]. Should these fail, lumbar or caudal epidural glucocorticoid injections can be administered for pain management. Surgery is indicated, in cases of pain refractory to conservative treatment or if neurological deficits develop.

New biological therapies are being investigated for use in LBP. One such treatment is the use of platelet-rich plasma (PRP). PRP is plasma containing concentrated platelets acquired from autologous blood. Advocates of PRP call it a bridge between conservative therapy and surgery. Platelets are rich in cytokines and growth factors and these are believed to accelerate the body's repair processes. They could, therefore, be beneficial for treating the inflamed nerves in lumbar radiculopathy and canal stenosis.

The purpose of this article is to provide a review of the currently available literature concerning the use of PRP in lumbar spine pathologies that lead to LBP. We hope this will prove useful to clinicians and academics interested in developing new therapies for LBP.

\section{What is Platelet-Rich Plasma?}

PRP is a sequestration of platelets in the plasma fraction of autologous blood, which when activated by an exogenous agent releases a host of mediators and growth factors [8-14]. Normal platelet counts in the blood range from $150,000 / \mu \mathrm{L}$ to $350,000 / \mu \mathrm{L}$. PRP, however may contain platelet concentrations of up to $1,000,000 / \mu \mathrm{L}$ [14]. It is this concentration in $5 \mathrm{~mL}$ of autologous plasma that is considered to be the practical definition of PRP [9]. However different concentrations and volumes are used in practice.

PRP was first used in dental implant procedures [9]. Its use since then has been extended to areas such as oral and maxillofacial surgery, cardiovascular surgery, spine surgery, plastic surgery, podiatric surgery, pulmonary care, and advanced wound care [10].

\section{Composition of Platelet-Rich Plasma}

Platelets are anucleate cell fragments derived from megakaryocytes in the bone marrow [10]. They contain organelles such as mitochondria, ribosomes, lysosomes, and two types of granules, alpha and dense.
Each platelet has about 50 to 80 alpha granules [10]. Alpha granules contain more than 300 different proteins [15]. Dense granules, on the other hand, are relatively few in number and contain important chemicals such as calcium, serotonin, histamine, and different phosphate compounds such as adenosine diphosphate, adenosine triphosphate, and guanosine diphosphate [16].

Platelets are a storehouse of important growth factors such as platelet-derived growth factor (PDGF), transforming growth factor-beta (TGF- $\beta$ ), insulin-like growth factor 1, vascular endothelial growth factorm and epithelial growth factor $[9,10,14,17]$. When released from degranulated platelets they mediate healing and play important roles in chemotaxis and migration, angiogenesis, and cell proliferation and differentiation. All these processes ultimately lead to repair [17]. There are also several cytokines, chemokines, and metabolites released by activated platelets that further supplement these actions [17].

Depending on the preparation technique, PRP may or may not contain leukocytes. Leukocytes are thought to release metalloproteinases and free radicals which may be counterproductive to the repair process and may also increase postinjection pain $[9,18]$. However, they may exert antimicrobial actions [19] and some researchers believe that leukocytes have crucial repair mediating enzymes as well [18].

\section{Preparation of Platelet-Rich Plasma}

Blood is first drawn from the patient at the time of treatment. An anticoagulant is used to prevent premature activation of the platelets [20]. The blood sample is centrifuged to separate the components into different layers one of which is formed by the PRP. The volume of PRP directly correlates to the baseline platelet count [21]. At this stage, one of two methods can be used to complete the preparation; the PRP method or the buffy coat method.

\section{Platelet-Rich Plasma Method}

Blood is initially centrifuged at a constant acceleration ("soft spin") to concentrate platelets in the supernatant. The platelet-containing plasma is then separated into a sterile tube and centrifuged at a higher speed ("hard spin") for the second time to acquire the platelet concentrate. This step leads to the creation of the PRP (lower one-third the platelet concentrate) and platelet-poor plasma (PPP) 
(the upper two-thirds of the platelet concentrate) [20].

\section{Buffy Coat Method}

The whole blood must initially be stored at a cool temperature (range, $20^{\circ} \mathrm{C}$ to $24^{\circ} \mathrm{C}$ ) before centrifugation [20]. High-speed centrifugation of the whole blood sample forms three layers; a superficial layer (PPP), an intermediate layer also called the "buffy coat" (platelets and white blood cells [WBCs]) and a deep layer consisting of red blood cells (RBCs). The buffy coat is then separated and centrifuged at a lower speed, yielding a layer of PRP, which is separated from the resulting solution [20,21].

\section{Classification of Platelet-Rich Plasma}

A standardized classification of PRP will increase the standardization of PRP reporting and the resultant data comparability. A wide range of classification systems have been recommended by different authors but the four main classification systems are mentioned below.

Mishra et al. [22] categorize PRP preparations into four main types depending on the concentration of PRP, the WBC content, and the activation status. Type 1 and type 2 PRP have WBC levels that are higher than the baseline. They differ in their activation status by an exogenous activator, such as calcium or thrombin. Type 2 is activated whereas type 1 is not. Similarly, type 3 and type 4 PRP have minimal WBCs and type 3 is not activated by an exogenous activator while type 4 is. Each group is then further subdivided based on the platelet concentration. If it is over 5 times higher than the baseline it is classed as subgroup A, if not, it is in subgroup B. Mishra's classification was acceptable for PRP preparations available in 2006 [13]. Mautner et al. [13], however, deem it insufficient as advancing technology, such as the development of double spin suspension systems, has allowed higher concentrations of platelets, with little or no neutrophils and RBCs in the PRP.

In 2009, Dohan Ehrenfest et al. [23,24] published another system, which classified PRP according to its platelet and leukocyte concentration and the absence or presence of fibrin. PRP was divided into pure PRP; leukocyte and PRP; pure platelet-rich fibrin (PRF); and leukocyte and PRF [23,24]. According to Mautner et al. [13], this approach is limited by its inability to be applied in nonoperative conditions due to the limited use of fibrin and the
Table 1. PLRA classification system proposed by Mautner et al. [13]

\begin{tabular}{|c|c|c|}
\hline PLRA classification & \multicolumn{2}{|c|}{ Criteria } \\
\hline P: platelets count & $\overline{\text { Volume injected }}^{P}$ & $\overline{\text { Cells/microlitter }}^{\mathrm{M}}$ \\
\hline \multirow[t]{2}{*}{ L: leukocyte content ${ }^{\text {a) }}$} & $>1 \%$ & + \\
\hline & $<1 \%$ & - \\
\hline \multirow[t]{2}{*}{ R: red blood cell content ${ }^{\text {b) }}$} & $>1 \%$ & + \\
\hline & $<1 \%$ & - \\
\hline \multirow[t]{2}{*}{ A: activation } & Yes & + \\
\hline & No & - \\
\hline
\end{tabular}

PLAR, platelet, leukocyte, red blood cells, and activation.

a) If white blood cells are present (+), the percentage of neutrophils should be reported. ${ }^{\text {bi }}$ The method of exogenous activation should be reported.

lack of information on RBC and WBC concentrations.

The "PAW" classification system was proposed by DeLong et al. [25] in 2012. This classification is based on the concentration of platelets, the activation status, and the $\mathrm{WBC}$ and neutrophil content relative to baseline [25]. The concentration of platelets ranged from P1 (baseline) to P4 (>1.2 million platelets $/ \mathrm{mL})$. Activation was either exogenous or nonexogenous and WBCs and neutrophils above or below baseline [25]. Mautner et al. [13], however, argue that this classification also failed to include RBCs and its categorization of WBCs and neutrophils into above and below baseline greatly underestimated their roles in PRP action.

In 2015, Mautner et al. [13] introduced their own classification system - the platelet, leukocyte, RBCs, and activation (PLRA) system. They classified PRP according to the following parameters [13]: (1) the concentration of platelets, the total number of platelets and injected volume; (2) the concentration of leukocytes; (3) the concentration of RBC; and (4) activation by exogenous agents. The features of the PLRA classification are summarized in Table 1.

\section{Intra Facet and Ligament Injections}

Facet joints have been implicated in $40 \%$ of cases of spine pain [6]. However, we found only three studies that investigated the results of PRP injections into the facet joints and the surrounding ligaments. Aufiero et al. [6] published a case series of five patients in 2015. PRP was injected bilaterally at multiple levels of the cervical spine of two patients. Two patients received PRP injections bilaterally in their lower lumbar levels and one patient had injections in the 
lower thoracic and upper lumbar levels. PRP was injected into the facet joints, capsule, supraspinous, and infraspinous ligaments under ultrasound or fluoroscopic guidance. All five cases reported a significant improvement in their pain. However, given the absence of a control group, it is not possible to conclude whether this treatment is better or worse than the established modalities.

Another study enrolled 19 patients with lumbar facet joint syndrome and injected autologous PRP into the lumbar facet joints under X-ray fluoroscopic guidance [26]. Pain control outcomes were measured using the Visual Analog Scale (VAS), Roland-Morris Disability Questionnaire (RMQ), Oswestry Disability Index (ODI), and modified MacNab criteria. Significant pain reduction was experienced by nine patients $(47.37 \%)$ immediately after treatment, $14(73.68 \%)$ at 1 week, and 15 patients $(78.95 \%)$ at 1 month, 2 months, and 3 months [26].

In 2017, a prospective comparative study randomized 46 patients into group A (intra-articular PRP) and group B (intra-articular local anesthetic and corticosteroid) [27]. Pain outcomes were measured using VAS, RMQ, ODI, and modified MacNab criteria. Both groups achieved statistically significant pain relief. Subjective satisfaction based on the modified MacNab criteria and the objective success rate for group B peaked (80\% and $85 \%$ ) after 1 month, but it was only $50 \%$ and $20 \%$ after 6 months. However, for group A, they increased over time. It was hence concluded that autologous PRP is a superior treatment option for longer duration efficacy [27]. This study addressed the previous two studies' lack of control group; however, it was limited by the small number of patients. More studies, with larger sample sizes and double-blinding, are needed for conclusive evidence.

\section{Epidural Injections}

Epidural steroid injections (ESIs) are an established method of pain management in lumbar radiculopathy. In fact, ESIs are the most widely performed pain management procedure in the world [28]. Their use has been documented and corroborated by more than 45 placebocontrolled trials and dozens of systematic reviews [28]. They may be injected trans foramen, intralaminar, or caudally via the sciatic hiatus. The trans foramen approach has shown to be the most efficacious as it is more targetspecific, has greater ventral epidural spread and is associated with lower chances of dural puncture [28].
Steroids work by inhibiting the arachidonic acid cascade thereby limiting inflammation of the lumbar nerve roots. Risk factors for this procedure include hemorrhage or hematoma formation, infection, paralysis, or spinal headache $[4,10]$. Steroid injections have also been implicated in spinal cord embolisms and septic and aseptic meningitis $[4,10]$.

The injection of PRP into the epidural space for lumbar radiculopathy is uncharted territory. In our literature search, we were able to find only two papers describing the injection of PRP in place of steroids.

The first study was a pilot study published in 2016 by Bhatia and Chopra [4]. Using the intralaminar approach, they injected $5 \mathrm{~mL}$ of PRP into the epidural spaces of 10 patients. Patients were followed up using the modified Oswestry Disability Questionnaire (MODQ), Straight Leg Raising Test (SLRT), and VAS scores. All patients showed sustained improvement up to the 3-month follow-up period. VAS scores for all patients were 4 or less except for one patient who scored 5. Most of the patients also showed an improvement in MODQ and SLRT to less than 30\% and greater than 70 , respectively.

The second paper was a case study published by Lemper et al. [10]. They report a 35-year-old female being chronically managed for neck pain and LBP with radiation into her arms and legs. She had a history of a motor vehicle collision, after which these symptoms arose. She had received multiple cervical and lumbar ESIs which gave her only a few weeks of relief before her pain returned. She had also undergone 6 months of physical therapy with no reported improvement in her symptoms.

She then became pregnant and the injections were abandoned as steroids can lead to pregnancy complications, prolonged labor, and in severe cases fetal and maternal death [10]. She agreed to epidural PRP injections and reported improvements in both her neck and lower back pain. There were no adverse effects of the treatment on the pregnancy and the patient delivered a healthy child.

Though both articles report positive results of epidural PRP injections, there is a need for further studies before wider use. Comparative studies with conventional therapies such as NSAIDs, physical therapy, and epidural steroids would help determine the efficacy of PRP compared to that of already established modalities.

A significant limitation of epidural PRP is that patients may not achieve acute pain relief. For this Bhatia and Chopra [4] suggest the supplementation of PRP with 
painkillers. Again, more studies are required to evaluate this.

\section{Intradiscal Injections}

An intervertebral disc (IVD) is a pad of cartilage between two adjacent vertebrae that acts as a shock absorber. It plays a vital role in stabilization as well as flexion and extension of the spine [29].

Being the largest avascular structure in an adult human, the IVD depends upon passive diffusion from the endplate vessels for nutrition. It is supplied by branches of the metaphyseal arteries around the outer annulus [30]. Since the IVD lacks a proper nutritional supply of growth factors, it has minimum self-repairing ability $[29,31]$.

Degenerative disc disease (DDD) involves the degeneration of one or more IVDs [32] and depends upon genetic and environmental factors [31]. The patient presents with pain that may radiate [32]. Current treatment options include conservative approaches such as anti-inflammatory drugs and physiotherapy, as well as spinal surgery [29]. Not only do current treatments completely ignore the underlying cause but treatment options like surgery restrict patient mobility and can interrupt their daily activity for nearly 6 weeks [32]. An alternative therapy like stem cells or growth factors might be a possible cheap solution to treat this pathology. It can be performed in outpatient facilities and takes very little time [5]. The purpose of intradiscal injections is to supply a high concentration of growth factors to poorly nourished IVD for growth and repair.

Several studies investigating the use of PRP on IVDs have been published [29-33] (Table 2). Obata et al. [31] induced disc degeneration in 12 rabbits by an annular puncture in two noncontiguous discs. This produced disc narrowing within 4 weeks. Autologous PRP or PPP were isolated from fresh blood. Releasate isolated from clotted PPP or PRP or phosphate-buffered saline (PBS) control was injected at the site of the degenerating disc. It was found that PRP releasate produced a statistically significant restoration of disc height. The PRP treatment group also had a higher number of chondrocyte-like cells. However, magnetic resonance imaging (MRI), T2 quantification did not show a statistical difference in mean T2 values of nucleus pulposus and annulus fibrosus among the three groups.

Another article demonstrates a similar finding. PRP was used with bone marrow-derived mesenchymal cells (BMSCs). A total of 30 rabbits were used in this experiment: 10 were in the PRP-BMSC group, 10 were injected with PRP or PBS, while another 10 were given no intervention and served as controls. MRI revealed the reparative effect of PRP in the PRP-BMSC group and PRP group. On the other hand, disc degeneration was unaffected in the PBS group. MRI results at 8 weeks demonstrated a statistically significant improvement in disc restoration in the PRPBMSC group as compared with other groups. The study, therefore, indicates better efficacy of PRP if used together with BMSCs [29].

Moving on to clinical studies, Comella et al. [32] report a study on 15 patients undergoing liposuction to extract mesenchymal cells. PRP was used with the adipose tissue for IVD injections. The results indicated a statistically significant improvement in flexion (pelvis, lumbar, and total flexion), pain, VAS, present pain intensity, and the scores of other questionnaires. There were no serious complications. ODI and Beck Depression Inventory scores showed improvement but with no statistical significance. The Dallas Pain Questionnaire showed an improvement in daily and work and leisure activities. However, there was an increase in anxiety and depression scores and a drop in social interest. The study, however, lacked a control group and further investigation is required. Levi et al. [33] state a similar finding in another prospective trial involving 22 patients with discogenic LBP who were treated with PRP IVD injections. The therapy was considered successful if the patients managed a $30 \%$ improvement in ODI and $50 \%$ improvement in VAS after the 1 month, 2 months, and 6 months following the injection. At 6 months, 47\% had a successful outcome in ODI and $41 \%$ had a successful outcome in VAS according to the criteria set. The trial showed encouraging preliminary data supporting the use of intradiscal PRP injections. This study also lacked a control group.

Another study conducted by Tuakli-Wosornu et al. [30] consisted of adult patients with chronic ( $\geq 6$ months), moderate-to-severe lumbar discogenic pain that was unresponsive to conservative treatment. The 58 study participants were randomized to receive intradiscal PRP or contrast agent after provocative discography. In the PRP group, 56\% were satisfied with the treatment compared with $17 \%$ of the control group. Those who received PRP maintained their Functional Rating Index for 1 year. There were no side effects or complications such as disc 


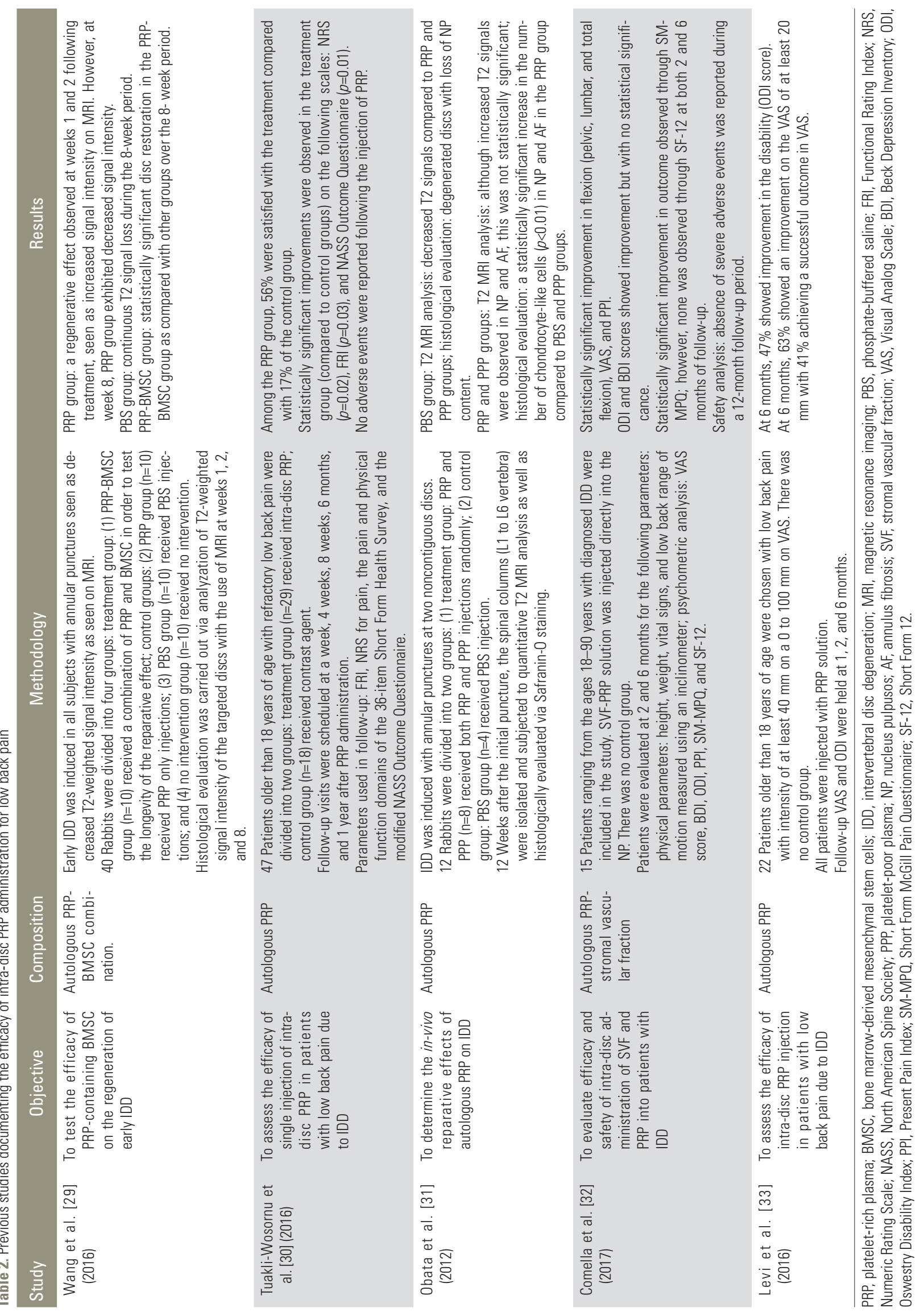


space infection, neurologic injury, or progressive herniation after receiving the PRP injection.

To summarize, intradiscal PRP injections can be a safe, cheap, and feasible treatment to counter IVD degeneration associated LBP. It is vital to administer PRP early in the course of the treatment to stimulate the growth of the remaining cells in the disc. If the treatment is delayed, the number of active cells in the disc will be at a minimum and the PRP will possibly fail to induce the desired impact [29]. Although intradiscal PRP injections show promising results, there is a need for more studies with larger sample sizes and adequate control groups. Further studies are also needed to define the subset of patients most likely to benefit from this treatment.

\section{Spinal Fusion}

Spinal fusion is a procedure used to treat chronic lumbar pain due to DDD [34]. The procedure involves joining the vertebrae, to eliminate movement between them, resulting in a continuous bone. The posterolateral fusion of two adjacent vertebrae is a frequently performed procedure on the lumbar spine $[34,35]$. Bone autograft extracted from the iliac spine is considered to be the gold standard [3436]. The rationale behind this procedure is that the graft has osteoinductive and osteoconductive properties and contains osteogenic cells that will result in new bone formation [36].

Harvesting the bone graft can result in serious complications such as donor morbidity, bleeding from the harvest site, nerve injury, and subsequent chronic pain and infection [34,36-38]. Moreover, the process requires a lot of time and may still result in inadequate availability of the graft [34,37].

The use of PRP in spinal fusion surgery could be a possible solution to this problem. TGF- $\beta$ and PDGF found in the alpha granules of the concentrated platelets encourage osteoblast proliferation [34,39-42]. They are a cheap and natural pool of growth factors that is available instantly in the operating theater $[34,37,40]$. They also promise faster recovery times [40]. Current data available in the literature demonstrate encouraging results.

Landi et al. [40] described 14 patients who underwent posterolateral fusion. In each patient, PRP along with autologous bone was used on one-half of the operative field and autologous bone graft alone on the other half. All patients underwent serial CT scans and plain X-rays at 3 and 6 months after surgery to evaluate bone fusion. At 3 months, the authors found an increased rate of fusion in the PRP operated field as compared to the one in which only the autologous graft was used. Although this difference was balanced out at 6 months, bone density in the PRP operated field remained higher [40].

A similar finding was reported by Tarantino et al. [34] in 20 patients who underwent posterolateral arthrodesis in lumbar spine surgery. In one hemifield, cancellous bone with PRP was used while in the other cancellous bone with saline was applied. The results after 6 months demonstrated a statistically significant increase in bone density and fusion rates in the PRP hemifield compared to the half in which cancellous bone alone was used.

Lowery et al. [43] reported a retrospective study of 19 patients with $100 \%$ fusion rates who underwent spinal fusion with the use of autologous growth factors (AGF) acquired from ultrafiltration of platelets, along with the bone graft and hydroxyapatite. Similarly, intertransverse lumbar fusion autograft alone was shown to produce less bone maturation in comparison with the addition of PRP to the autograft [44].

Some articles, however, report that PRP has no use in spinal fusion and some even describe negative outcomes. Sys et al. [41] investigated the effects of PRP in monosegmental posterior lumbar interbody fusion in 40 patients. The VAS score and ODI differences between the "allograft with PRP" group and "allograft only" group were statistically insignificant. Another article demonstrated the inhibitory effects of AGF used in conjunction with iliac crest grafts in posterolateral spinal fusion surgery [45].

\section{Intramuscular Injections}

The lumbar multifidus (LMF) muscle stabilizes the spine because of its morphology and its anatomical position. Its fibers are short with a high cross-sectional area which supports the muscle's role in spine stabilization rather than motion. The relation between lower back pain and the multifidus muscle has been well established by several studies [46]. In chronic LBP with monosegmental disc degeneration, the size and cross-sectional area of the muscle decreases [46].

We found one study that reports the role of platelet leukocyte rich plasma (PLRP) injections on LMF muscle [46]. PLRP intramuscular injections were given to 115 patients and the outcomes were monitored using the Numeric Rat- 
ing Scale and ODI. Both scores showed a statistically significant improvement. The overall successful outcome was $71.2 \%$, thus demonstrating that PLRP injections in the LMF muscle can possibly prove to be a safer and cheaper approach to treat LBP. The lack of a control group, however, limits further decisive conclusions.

\section{Limitations of Platelet-Rich Plasma}

The premature release of growth factors through degranulation, the fact that growth factors are released for a shorter duration of time after activation, and their susceptibility to proteolytic enzymes may render PRP ineffective [35,37]. Variable concentrations and quality of PRP because of the differences between individuals also limit its use and contributes to some of the negative results published. Lastly, the optimal time for PRP implantation, the optimal concentration of platelets in the PRP sample and whether PRP should be used in the inactivated or activated form is yet to be established $[35,39]$.

\section{Conclusions}

In conclusion, the use of PRP in various injections, such as intrafacet, intraligament, epidural, intradiscal, spinal fusion, and intramuscular injections, has yielded promising results that have been reported in recent literature. However, further studies are required with larger sample sizes and control groups to prove its efficacy and establish its routine use in surgery. The stage of LBP at which PRP provides the most advantage and how PRP injections perform in comparison to conservative measures such as NSAIDS and physiotherapy are also areas for further investigation.

\section{Conflict of Interest}

No potential conflict of interest relevant to this article was reported.

\section{References}

1. Soliman AF, Hammad GA, El-gamal RI, Al-Rabiei MA. Assessment of the implication of epidural steroid injection versus other conservative measures in the management of lumbar disc herniation. Egypt Rheumatol Rehabil 2016;43:53-8.
2. Knezevic NN, Mandalia S, Raasch J, Knezevic I, Candido KD. Treatment of chronic low back pain: new approaches on the horizon. J Pain Res 2017;10:111123.

3. Chopra G, Srivastav N. To evaluate the comparative efficacy of various drug combination via epidural route in treatment of pain in patients of intervertebral disc herniation. IOSR J Dent Med Sci 2014;13:76-9.

4. Bhatia R, Chopra G. Efficacy of platelet rich plasma via lumbar epidural route in chronic prolapsed intervertebral disc patients: a pilot study. J Clin Diagn Res 2016;10:UC05-7.

5. Monfett M, Harrison J, Boachie-Adjei K, Lutz G. Intradiscal platelet-rich plasma (PRP) injections for discogenic low back pain: an update. Int Orthop 2016;40:1321-8.

6. Aufiero D, Vincent H, Sampson S, Bodor M. Regenerative injection treatment in the spine: review and case series with platelet rich plasma. J Stem Cells Res Rev Rep 2015;2:1019.

7. Mietsch A, Neidlinger-Wilke C, Schrezenmeier H, et al. Evaluation of platelet-rich plasma and hydrostatic pressure regarding cell differentiation in nucleus pulposus tissue engineering. J Tissue Eng Regen Med 2013;7:244-52.

8. Cho AR, Kim HK, Kwon JY, Kim TK, Choi YM, Kim $\mathrm{KH}$. The incorporation of platelet-rich plasma into calcium phosphate cement enhances bone regeneration in osteoporosis. Pain Physician 2014;17:E737-45.

9. Ornetti P, Nourissat G, Berenbaum F, et al. Does platelet-rich plasma have a role in the treatment of osteoarthritis? Joint Bone Spine 2016;83:31-6.

10. Lemper BA, Rhodes S, Njoroge BK, Yurgelon JT, Klassen LJ. Chronic pain management and pregnancy: a platelet rich plasma epidural case study: Lemper research and development [Internet]. Denver (CO): The American Academy/Association of Orthopedic Medicine [cited 2019 Apr 20]. Available from: http:// www.aaomed.org/AAOM/files/ccLibraryFiles/Filename/000000000115/PRP\%20Case\%20Study\%20 -\%20Pregnancy.pdf.

11. Gui K, Ren W, Yu Y, Li X, Dong J, Yin W. Inhibitory effects of platelet-rich plasma on intervertebral disc degeneration: a preclinical study in a rabbit model. Med Sci Monit 2015;21:1368-75.

12. Sheth U, Simunovic N, Klein G, et al. Efficacy of autologous platelet-rich plasma use for orthopaedic 
indications: a meta-analysis. J Bone Joint Surg Am 2012;94:298-307.

13. Mautner K, Malanga GA, Smith J, et al. A call for a standard classification system for future biologic research: the rationale for new PRP nomenclature. PM R 2015;7(4 Suppl):S53-9.

14. Marx RE. Platelet-rich plasma: evidence to support its use. J Oral Maxillofac Surg 2004;62:489-96.

15. Coppinger JA, Cagney G, Toomey S, et al. Characterization of the proteins released from activated platelets leads to localization of novel platelet proteins in human atherosclerotic lesions. Blood 2004;103:2096104.

16. Rendu F, Brohard-Bohn B. The platelet release reaction: granules' constituents, secretion and functions. Platelets 2001;12:261-73.

17. Navani A, Li G, Chrystal J. Platelet rich plasma in musculoskeletal pathology: a necessary rescue or a lost cause? Pain Physician 2017;20:E345-56.

18. Rayegani SM, Raeissadat SA, Taheri MS, et al. Does intra articular platelet rich plasma injection improve function, pain and quality of life in patients with osteoarthritis of the knee?: a randomized clinical trial. Orthop Rev (Pavia) 2014;6:5405.

19. Cieslik-Bielecka A, Gazdzik TS, Bielecki TM, Cieslik T. Why the platelet-rich gel has antimicrobial activity? Oral Surg Oral Med Oral Pathol Oral Radiol Endod 2007;103:303-5.

20. Dhurat R, Sukesh M. Principles and methods of preparation of platelet-rich plasma: a review and author's perspective. J Cutan Aesthet Surg 2014;7:18997.

21. Gulliksson H. Platelets from platelet-rich-plasma versus buffy-coat-derived platelets: what is the difference? Rev Bras Hematol Hemoter 2012;34:76-7.

22. Mishra A, Harmon K, Woodall J, Vieira A. Sports medicine applications of platelet rich plasma. Curr Pharm Biotechnol 2012;13:1185-95.

23. Dohan Ehrenfest DM, Bielecki T, Mishra A, et al. In search of a consensus terminology in the field of platelet concentrates for surgical use: platelet-rich plasma (PRP), platelet-rich fibrin (PRF), fibrin gel polymerization and leukocytes. Curr Pharm Biotechnol 2012;13:1131-7.

24. Dohan Ehrenfest DM, Rasmusson L, Albrektsson T. Classification of platelet concentrates: from pure platelet-rich plasma (P-PRP) to leucocyte- and platelet-rich fibrin (L-PRF). Trends Biotechnol 2009;27:158-67.

25. DeLong JM, Russell RP, Mazzocca AD. Platelet-rich plasma: the PAW classification system. Arthroscopy 2012;28:998-1009.

26. Wu J, Du Z, Lv Y, et al. A new technique for the treatment of lumbar facet joint syndrome using intra-articular injection with autologous platelet rich plasma. Pain Physician 2016;19:617-25.

27. Wu J, Zhou J, Liu C, et al. A prospective study comparing platelet-rich plasma and local anesthetic (LA)/ corticosteroid in intra-articular injection for the treatment of lumbar facet joint syndrome. Pain Pract 2017;17:914-24.

28. Cohen SP, Bicket MC, Jamison D, Wilkinson I, Rathmell JP. Epidural steroids: a comprehensive, evidencebased review. Reg Anesth Pain Med 2013;38:175-200.

29. Wang SZ, Jin JY, Guo YD, et al. Intervertebral disc regeneration using platelet-rich plasma-containing bone marrow-derived mesenchymal stem cells: a preliminary investigation. Mol Med Rep 2016;13:347581.

30. Tuakli-Wosornu YA, Terry A, Boachie-Adjei K, et al. Lumbar intradiskal platelet-rich plasma (PRP) injections: a prospective, double-blind, randomized controlled study. PM R 2016;8:1-10.

31. Obata S, Akeda K, Imanishi T, et al. Effect of autologous platelet-rich plasma-releasate on intervertebral disc degeneration in the rabbit anular puncture model: a preclinical study. Arthritis Res Ther 2012;14:R241.

32. Comella K, Silbert R, Parlo M. Effects of the intradiscal implantation of stromal vascular fraction plus platelet rich plasma in patients with degenerative disc disease. J Transl Med 2017;15:12.

33. Levi D, Horn S, Tyszko S, Levin J, Hecht-Leavitt C, Walko E. Intradiscal platelet-rich plasma injection for chronic discogenic low back pain: preliminary results from a prospective trial. Pain Med 2016;17:1010-22.

34. Tarantino R, Donnarumma P, Mancarella C, et al. Posterolateral arthrodesis in lumbar spine surgery using autologous platelet-rich plasma and cancellous bone substitute: an osteoinductive and osteoconductive effect. Global Spine J 2014;4:137-42.

35. Kamoda H, Ohtori S, Ishikawa T, et al. The effect of platelet-rich plasma on posterolateral lumbar fusion in a rat model. J Bone Joint Surg Am 2013;95:1109- 
16.

36. Zakaria Z, Seman CN, Buyong Z, Sharifudin MA, Zulkifly AH, Khalid KA. Histological evaluation of hydroxyapatite granules with and without plateletrich plasma versus an autologous bone graft: comparative study of biomaterials used for spinal fusion in a New Zealand white rabbit model. Sultan Qaboos Univ Med J 2016;16:e422-9.

37. Scholz M, Schleicher P, Eindorf T, et al. Cages augmented with mineralized collagen and platelet-rich plasma as an osteoconductive/inductive combination for interbody fusion. Spine (Phila Pa 1976) 2010;35:740-6.

38. Kamoda H, Yamashita M, Ishikawa T, et al. Plateletrich plasma combined with hydroxyapatite for lumbar interbody fusion promoted bone formation and decreased an inflammatory pain neuropeptide in rats. Spine (Phila Pa 1976) 2012;37:1727-33.

39. Cinotti G, Corsi A, Sacchetti B, Riminucci M, Bianco P, Giannicola G. Bone ingrowth and vascular supply in experimental spinal fusion with platelet-rich plasma. Spine (Phila Pa 1976) 2013;38:385-91.

40. Landi A, Tarantino R, Marotta N, et al. The use of platelet gel in postero-lateral fusion: preliminary re- sults in a series of 14 cases. Eur Spine J 2011;20 Suppl 1:S61-7.

41. Sys J, Weyler J, Van Der Zijden T, Parizel P, Michielsen J. Platelet-rich plasma in mono-segmental posterior lumbar interbody fusion. Eur Spine J 2011;20:1650-7.

42. Bose B, Balzarini MA. Bone graft gel: autologous growth factors used with autograft bone for lumbar spine fusions. Adv Ther 2002;19:170-5.

43. Lowery GL, Kulkarni S, Pennisi AE. Use of autologous growth factors in lumbar spinal fusion. Bone 1999;25(2 Suppl):47S-50S.

44. Sethi PM, Miranda JJ, Kadiyala S, et al. Evaluation of autologous platelet concentrate for intertransverse process lumbar fusion. Am J Orthop (Belle Mead NJ) 2008;37:E84-90.

45. Weiner BK, Walker M. Efficacy of autologous growth factors in lumbar intertransverse fusions. Spine (Phila Pa 1976) 2003;28:1968-71.

46. Hussein M, Hussein T. Effect of autologous platelet leukocyte rich plasma injections on atrophied lumbar multifidus muscle in low back pain patients with monosegmental degenerative disc disease. SICOT J 2016;2:12. 